\title{
L'ascolto come strumento relazionale in ambito dialitico: indagine conoscitiva
}

\author{
Maria Pia Zito
}

Infermiere Case Manager, Nefrologia, Dialisi e Trapianto, Servizio di Day Service Peritoneale, Direttore Prof. S. Stefoni, Ospedale S. Orsola, Bologna

\begin{abstract}
The "ABILITY TO LISTEN" AS AN INSTRUMENT IN DIALYSIS: A SURVEY
Abstract. Understanding the needs of who is in front of us, welcoming, accepting and guiding people that asks for help, being authentic, able to listen and understanding are all skills and abilities needed for any counseling professional who chose to follow this difficult and challenging career path. In this article, we will examine the relationship between the nurse and the patient in the field of dialysis. The study was conducted from May 28 to June 15, 2012 by distributing a questionnaire to uremic patients and nursing staff working at the Operative Unit of Hemodialysis, peritoneal dialysis and pre-dialysis of the S. Orsola Hospital in Bologna.

Aims of the research:

- verify the existence and the degree of an educational communicative relationship between patients and nurses;

- determine if the quality of the communication is equally perceived by both nurses and patients;

- determine if the specific communication induced beneficial effects on the psychology of the patients; specifically, if it enabled the patient to face the social, psychological and domestic difficulties determined by the disease and its treatment in daily life.
\end{abstract}

Key words: Counseling, Empathy, Helping relationship, Dialysis patient

Conflict of interest: None.

Ricevuto: 30 Maggio 2013; Accettato: 29 Luglio 2013

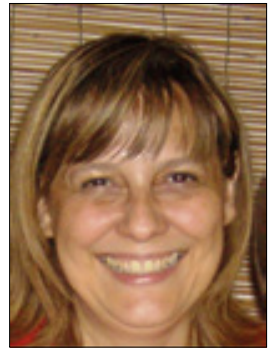

Maria Pia Zito

\section{Introduzione}

Oggi si comunica sempre meno ed è sempre più difficile farlo in modo efficace. Le incomprensioni e i malintesi sono la causa della fine di tanti rapporti. In famiglia e nei luoghi di lavoro ci si sente sempre più soli e le relazioni diventano sempre più fragili. La capacità d'ascolto, la comprensione, l'accettazione, l'empatia e la capacità di confrontarsi, aprendo se stesso all'altro, diventano qualità sempre più rare, malgrado la nostra sia la società della comunicazione, dove a dominare sono i riflettori, i microfoni, i media... e dove il linguaggio è spesso solo uno slogan.

Siamo condizionati da un modello sociale narcisistico, in cui dirompe fortemente il bisogno di imporsi e di affermare il successo, valorizzando l'iperattivismo e la capacità di trovare soluzioni veloci a problemi contingenti invece di dare spazio alla dimensione dell'interiorità, che richiede un tempo più ampio per la riflessione e la ricettività. La comunicazione è una strada a due sensi dove il solo udire è un atto fisico, mentre l'ascoltare è un'azione intellettuale ed emotiva.

\section{Ascolto e Comunicazione}

L'arte di ascoltare è innata in certe persone, ma totalmente assente in altre. Ascoltare non significa solo "udire", ma riflettere e concedere all'altro del tempo per immedesimarsi, per capire e per, eventualmente, aiutare. Ascoltare significa tenere in considerazione e dimostrare stima e rispetto. Quando, in una conversazione, l'interlocutore si distrae, si guarda in giro e perde il filo, non è solo la delusione di non essere ascoltati che dà fastidio, ma il pensiero di non suscitare nessunissimo interesse. Ascoltare vuol dire fare silenzio dentro di noi e far tacere le tante parole che stigmatizzano, che interpretano e che, a tutti i costi, vogliono trovare soluzioni veloci: solo da questo silenzio può nascere l'ascolto dell'altro. Dialogo è, allora, prima di tutto imparare un'arte che ci permette di capire e accettare l'altro, ma che aiuta nello stesso tempo l'altro a capire e a costruire pian piano la fiducia di cui si ha bisogno per un vero confronto: è un processo lento che richiede pazien- 
za e umiltà. In qualsiasi contesto educativo, assistenziale o terapeutico l'esperienza relazionale risulta coinvolgente più che impartire nozioni e offrire rifugi occasionali o risposte puramente efficienti. La comunicazione non è semplicemente parlare, ma presuppone necessariamente una relazione e, quindi, uno scambio.

L'ascolto che si nutre solo di tecnica diventa irrimediabilmente opaco e distante; per questo chi lavora nella relazione di aiuto ha chiara la sua importanza. Non è così semplice imparare ad ascoltare. Non basta essere accoglienti e caldi, ma occorre concentrarsi sui bisogni della persona, liberandosi dalla necessità urgente di controllare la relazione, di giudicare e di dare spazio al senso di onnipotenza. Capire i bisogni di chi ci sta di fronte, accogliere, accettare ed essere autentici e saper ascoltare, comprendere e orientare le persone che chiedono aiuto sono capacità e abilità che si distinguono in chi fa counseling e ha scelto di percorrere una strada professionale difficile e impegnativa.

\section{Fasi comunicative nell'assistenza infermieristica (1)}

Fondamentale in ogni progetto di aiuto è, quindi, una corretta comunicazione e, in questo caso, esamineremo quella tra infermiere e paziente.

Le fasi comunicative nell'assistenza infermieristica possono essere riassunte in tre momenti:

Apertura: si sviluppa al momento del contatto tra infermiere e paziente; è, con ogni probabilità, la fase più delicata, dato che è in grado di condizionare la qualità del rapporto comunicativo.

Si tratta della fase di accoglienza, preliminare alla relazione vera e propria; a seconda di come venga condotta questa fase, il paziente si sente riconosciuto come persona nella sua globalità anziché come caso clinico.

La considerazione della persona da parte dell'infermiere si esprime con un clima di disponibilità e sicurezza, nella quale possa sentirsi a proprio agio e trattato con dignità; è, inoltre, necessaria una serie di attenzioni, come quella di informare le persone appena arrivate su caratteristiche ambientali, orari e abitudini.

L'accoglienza introduce alla conoscenza reciproca, che si verifica in una dinamica interpersonale appena avviata e il cui avvicinarsi avviene gradatamente, man mano che la relazione evolve e matura.

L'accoglienza non va intesa fine a se stessa ma come momento introduttivo della relazione vera e propria; essa permette a entrambi gli interlocutori di abituarsi alla presenza fisica dell'altro e al suo modo di comunicare con le parole e con il linguaggio non verbale.

L'elemento fondamentale di questa fase è l'ascolto.

Peraltro, l'infermiere ha la possibilità di raccogliere un numero di dati personali oggettivi e soggettivi, filtrati attraverso la percezione che il paziente ha della situazione che lo riguarda, la quale è in funzione di fattori di tipo culturale e sociale, legati all'esperienza.

Operativa: quando l'infermiere ritiene perfezionato l'accordo con il malato, dirige l'interazione verso la fase operativa, dando inizio al processo di aiuto vero e proprio. È in questa fase che si verifica l'azione di aiuto all'assistito. Il processo di aiuto centrato sul malato, nel corso del quale lo si aiuta a risolvere i problemi, favorisce in lui la stima e l'indipendenza.

Conclusiva: il distacco dagli operatori sanitari, al termine dell'assistenza, vuol dire dimissione o morte, ma, nel nostro caso del paziente dializzato, possiamo parlare anche di trapianto e di situazione traumatica e non prevedibile nel tempo. Nel caso del trapianto, la persona è emotivamente confusa perché se, da un lato, è fiduciosa e serena nella prospettiva di poter tornare a vivere "normalmente", dall'altra dovrà re-inventarsi la propria esistenza e tutte le certezze che la malattia ha fatto cambiare. Il compito dell'infermiere è di rassicurare il paziente e di trasmettere più dati possibili su ciò che gli accadrà e su come la sua vita cambierà.

\section{La relazione di aiuto}

Una relazione di aiuto presuppone l'esistenza di due persone, delle quali una ha un problema che la blocca, creando una situazione di disagio che le impedisce di vivere serenamente, mentre l'altra cerca, tramite l'uso di tecniche comunicative mirate, di aiutare il soggetto in difficoltà a risolvere il suo stato di sofferenza. Il fine è l'utilizzo del dialogo come terapia.

Caratteristiche peculiari di una relazione di aiuto sono: volontà reciproca, da parte del terapeuta e del paziente, di relazionarsi e l'esistenza di un rapporto comunicativo tale da permettere l'instaurarsi di un clima di fiducia.

Lo scopo della relazione di aiuto è di permettere al paziente una migliore conoscenza di sé, di renderlo in grado di affrontare il problema che è motivo di malessere psichico nella maniera a lui più congeniale, aiutandolo ad acquisire capacità decisive non emotivamente influenzate dalla malattia, e, non da ultimo, la possibilità di aiutarlo a ricercare una motivazione per vivere in modo soddisfacente nonostante le limitazioni imposte dalla patologia (2).

Altri contenuti indispensabili da attivare sono:

- Far percepire la malattia come un evento di crescita individuale e non come un ripiego. Lasciare che il paziente racconti il proprio vissuto e le proprie esperienze, esprimendo ciò che "è veramente", con le proprie sensazioni e paure.

- Stimolare la presa di coscienza rispetto alla propria situazione per poterla gestire.

- Aiutare la persona ad adattarsi alla situazione che sta vivendo, senza ricercare in altri la colpa della sua infermità e del disagio psicologico conseguentemente generato.

- Aiutare la persona a discriminare gli obiettivi realizzabili da quelli impossibili; fra i primi distinguere quelli attuabili nel breve termine (p. es., riprendere la comunicazione con dei familiari allontanati), piuttosto che nel lungo termine (p. es., superare un disagio psicologico). Gli obiettivi devono essere chiari come pure i mezzi a disposizione per realizzarli.

- Riattivare e riorganizzare le risorse originarie psicologiche dell'assistito, rendendolo capace di azioni compatibili con il suo vero modo di essere e rendendolo più accettante, sereno 
e socialmente integrato.

- Metterlo in condizione di arrivare da solo a percepire ciò che sente e di verbalizzarlo consciamente (Monformoso 2001).

\section{Quesiti di ricerca}

L'infermiere, arricchendosi di un bagaglio culturale specifico, può migliorare la qualità di vita dell'assistito attraverso un'appropriata relazione comunicativa? L'infermiere, con il proprio ascolto empatico, può aiutare la persona a prendere serena coscienza della propria condizione trasformando il "dis-agio", riferito al proprio vissuto di persona sana, in "agio" nella sua nuova dimensione esistenziale?

Volendo verificare lo stato della comunicazione percepita in un grande servizio di dialisi è stata intrapresa una breve inchiesta condotta con la somministrazione di un questionario ai malati uremici e al personale infermieristico operante nell'Unità Operativa di Emodialisi Predialisi e Dialisi Peritoneale dell'Ospedale S. Orsola diretto dal Direttore Prof. S. Stefoni (3).

\section{Obiettivi}

Gli obiettivi primari della ricerca intrapresa sono stati:

A. Verificare l'esistenza di un rapporto comunicativo educativo tra malati e infermieri.

B. Stabilire se il livello qualitativo è percepito allo stesso modo sia dagli infermieri che dai pazienti.

C. Verificare se la comunicazione instaurata abbia un effetto benefico sulla psicologia del paziente, tale da permettergli di affrontare e superare eventuali disagi sociali, psicologici e familiari apportati dalla malattia e dal trattamento.

Obiettivo secondario è stato quello di individuare aree tematiche che potrebbero essere spunto per successive ricerche di approfondimento sulla metodologia d'approccio relazionale per il sostegno del paziente nei luoghi di cura.

\section{Materiali e metodi}

L'indagine è stata condotta dal 28 Maggio al 15 Giugno 2012 presso il servizio emodialisi e dialisi peritoneale dell'Azienda Ospedaliera S. Orsola. Il tipo di rilevazione effettuata è stato occasionale, dal momento che ha raccolto $\mathrm{i}$ pareri degli intervistati solo in un preciso momento storico.

Lo strumento di indagine è stato il questionario strutturato a domande chiuse. Sono stati redatti due questionari speculari composti da 7 domande per i pazienti e da 9 per gli infermieri.

Sono stati distribuiti 38 questionari al personale infermieristico di cui sono stati restituiti 22 (pari al 57, 89\%, di cui 17 in emodialisi e 5 in dialisi peritoneale). Sono stati contattati 80 pazienti tra emodialisi, dialisi e predialisi e il $100 \%$ dei soggetti ha aderito volontariamente alla raccolta dei dati.

\section{Dati dei Pazienti Intervistati}

\begin{tabular}{|l|c|}
\hline \multicolumn{2}{|c|}{ PAZIENTI INTERVISTATI: 80 DI CUI } \\
\hline MASCHI & $35(35 \%)$ \\
\hline FEMMINE & $45(45 \%)$ \\
\hline TOTALE & $80(100 \%)$ \\
\hline
\end{tabular}

\begin{tabular}{|l|c|}
\hline \multicolumn{2}{|c|}{ ANNI DI DIALISI DEI PAZIENTI } \\
\hline DA 0 A 2 ANNI & $45(45 \%)$ \\
\hline DA 3 A 10 ANNI & $31(31 \%)$ \\
\hline PIÙ DI 10 ANNI & $4(4 \%)$ \\
\hline
\end{tabular}

\section{Dati dei Infermieri Intervistati}

\begin{tabular}{|l|c|}
\hline \multicolumn{2}{|c|}{ ANNI DI LAVORO IN DIALISI } \\
\hline DA 0 A 2 ANNI & 2 \\
\hline DA 3 A 10 ANNI & 8 \\
\hline PIÙ DI 10 ANNI & 12 \\
\hline \multicolumn{2}{|c|}{ INFERMIERI INTERVISTATI: 22 DI CUI } \\
\hline MASCHI & 5 \\
\hline FEMMINE & 17 \\
\hline TOTALE & 22 \\
\hline
\end{tabular}

\section{Analisi dei dati}

Rappresentazione grafica delle risposte ottenute con i questionari somministrati agli infermieri di emodialisi e dialisi peritoneale:

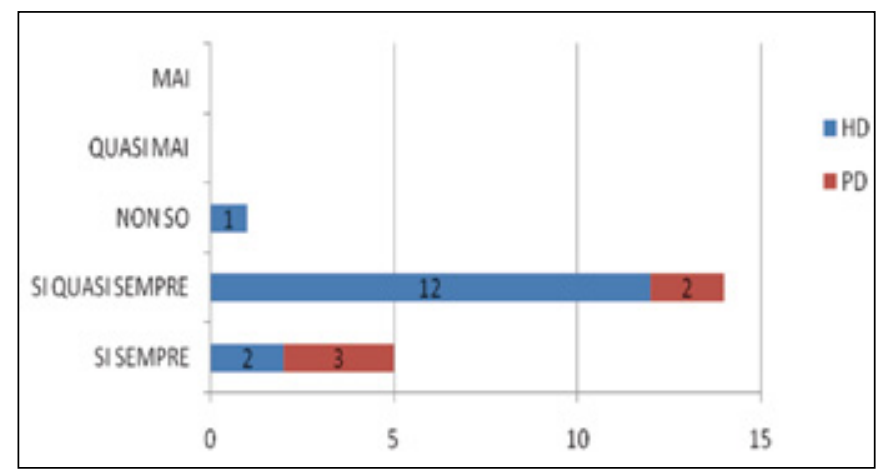

Fig. 1 - Penso di essere sempre chiaro e comprensibile nel dare le informazioni al paziente sulla sua malattia e sul suo trattamento? 


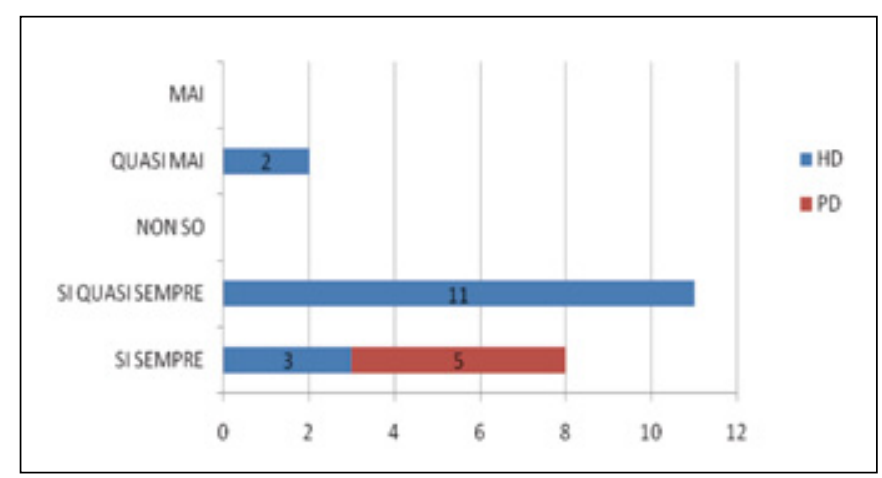

Fig. 2 - La persona assistita si informa regolarmente sull'esito degli esami eseguiti durante la dialisi?

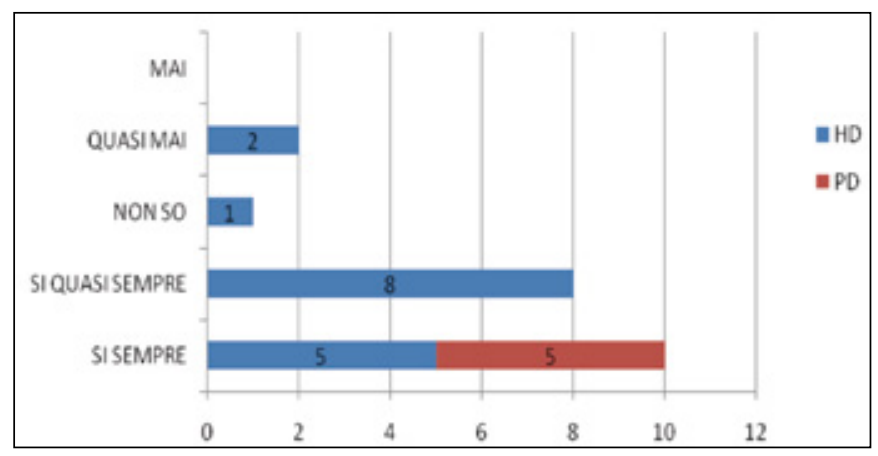

Fig. 4 - Quando il paziente mi parla della sua condizione di dializzato, riesco a non distrarmi e a rimanere concentrato sull'ascolto?

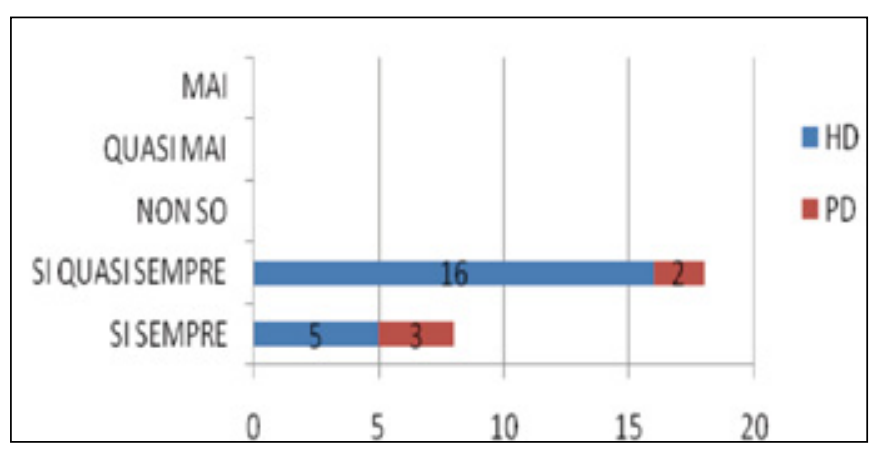

Fig. 6 - Ritengo che la comunicazione con i pazienti sia adeguata alla loro capacità di comprensione?

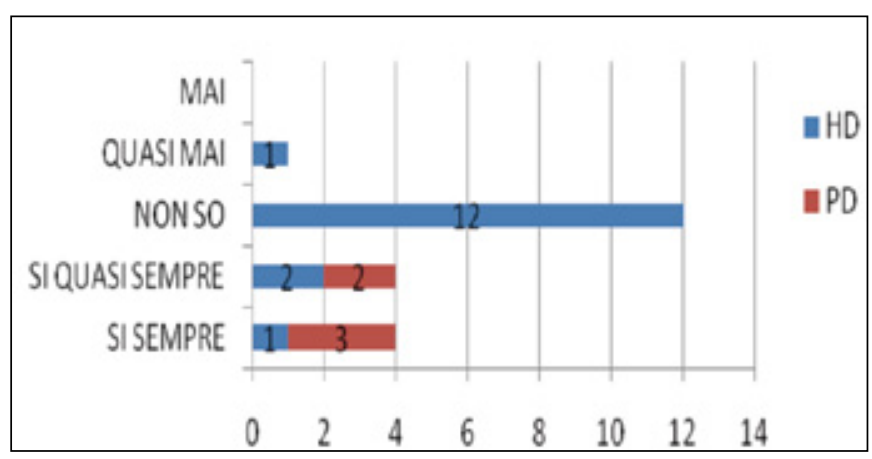

Fig. 8 - Penso di essere sempre in grado di gestire la relazione comunicativa con il paziente in modo sufficientemente adeguato e di evitare un eccessivo coinvolgimento emotivo?

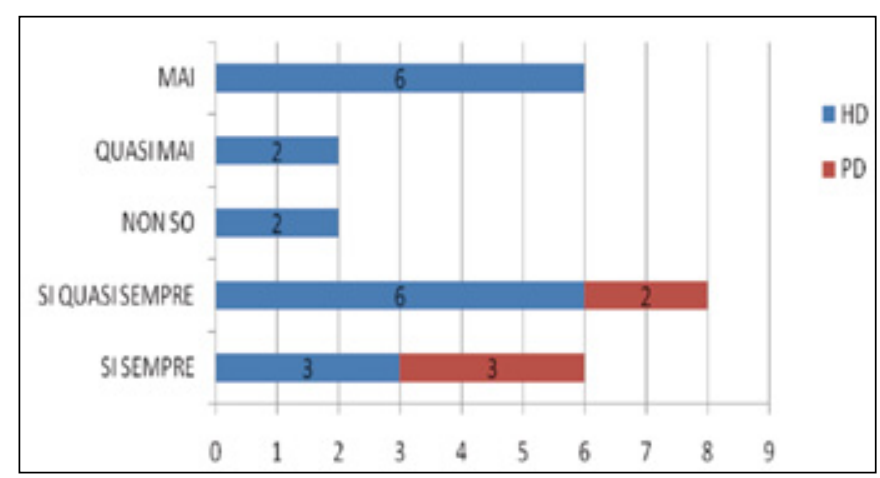

Fig. 3 - Quando il paziente mi parla delle sue problematiche relative al suo stato di salute esiste un luogo idoneo al colloquio?

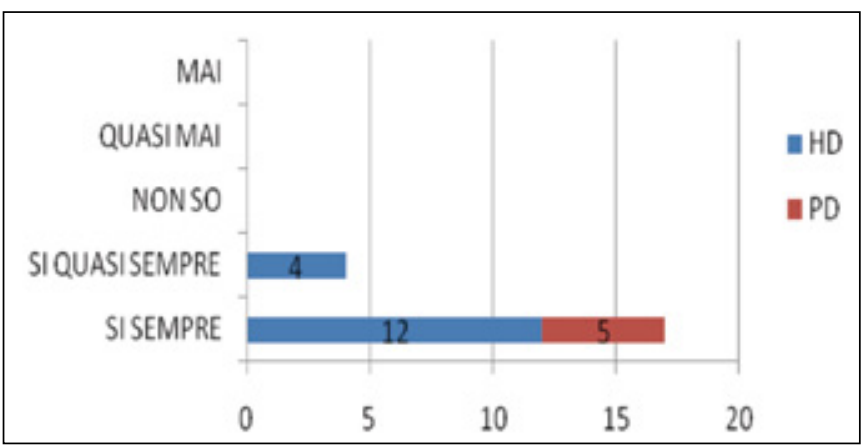

Fig. 5 - Quando fornisco informazioni mi accerto che il paziente abbia capito?

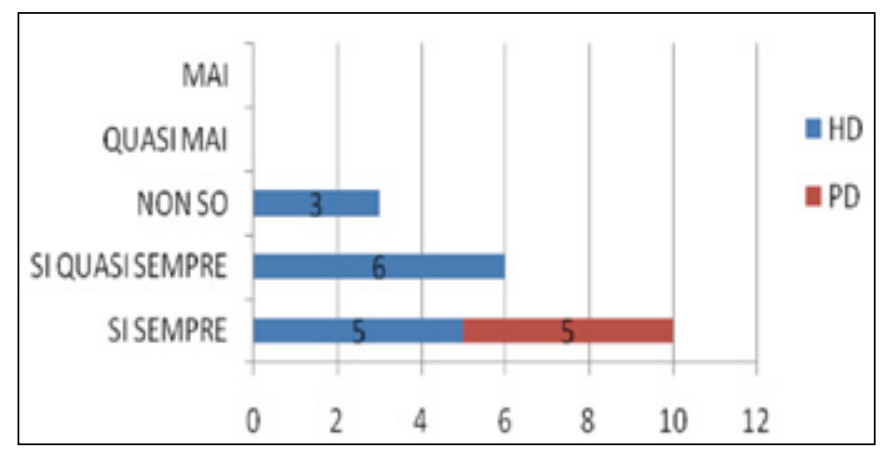

Fig. 7 - Quando devo comunicare al malato notizie di un certo impatto emotivo rispetto la sua privacy?

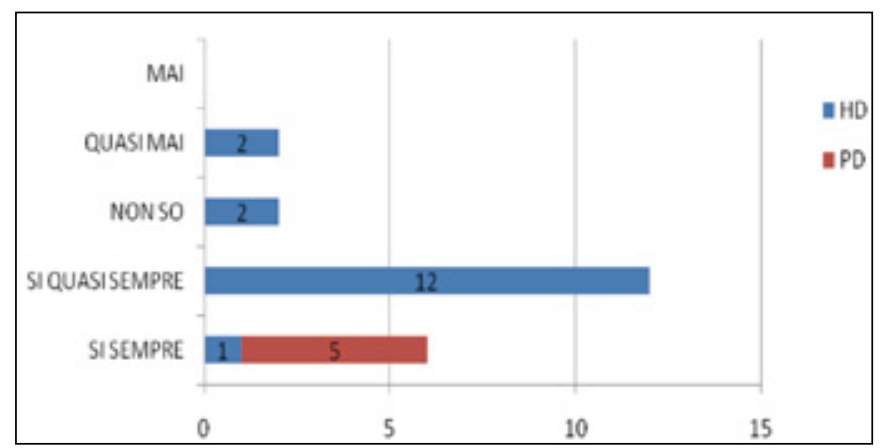

Fig. 9 - Ogni volta che un paziente richiede informazioni circa il suo stato di salute ho la possibilità di verificare ciò che ha capito? 
Rappresentazione grafica delle risposte ottenute con $i$ questionari somministrati ai pazienti in emodialisi, dialisi peritoneale e predialisi:

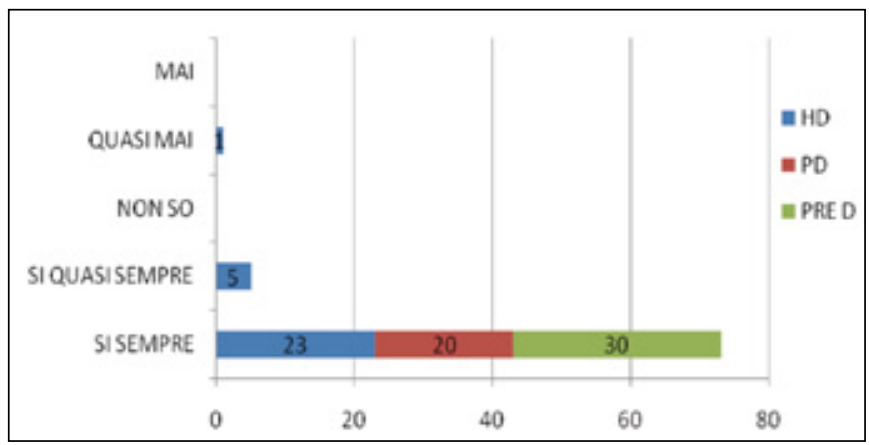

Fig. 2 - Quando chiede l'esito degli esami eseguiti durante la visita, l'infermiere è chiaro ed esaustivo nel fornire le informazioni?

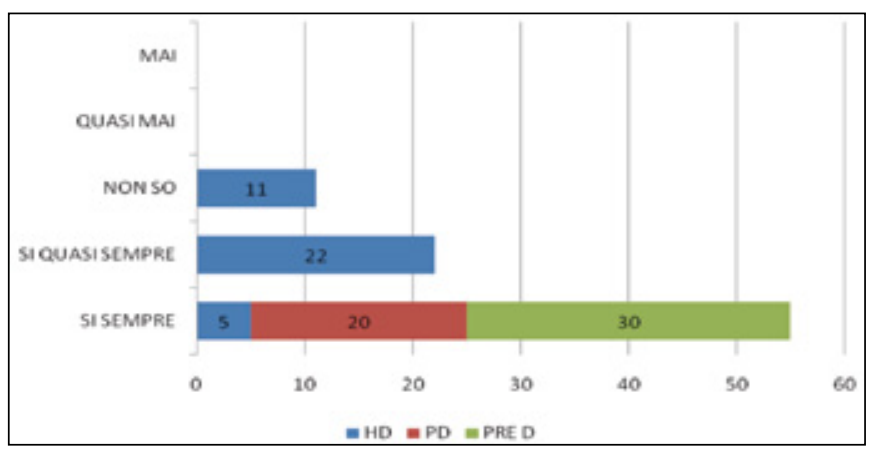

Fig. 4 - Quando le vengono date delle informazioni, l'infermiere si accerta che lei abbia capito?

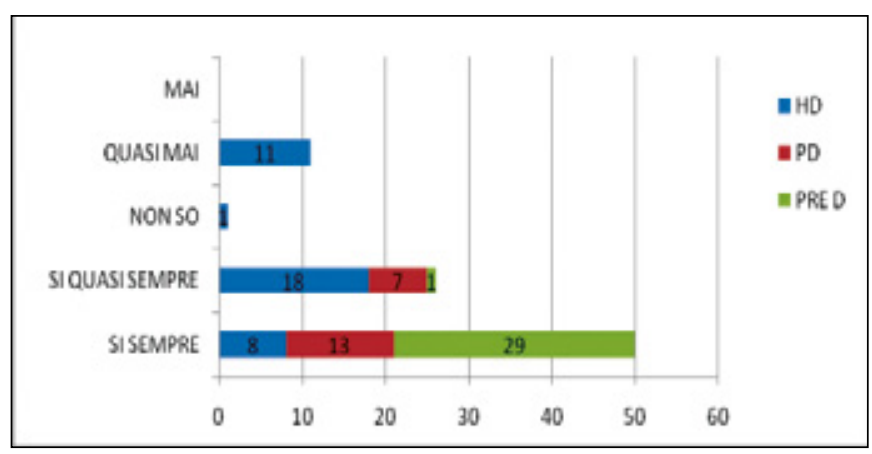

Fig. 6 - Quando l'infermiere comunica notizie di un certo impatto emotivo vi è sempre la possibilità di rispettare la privacy?

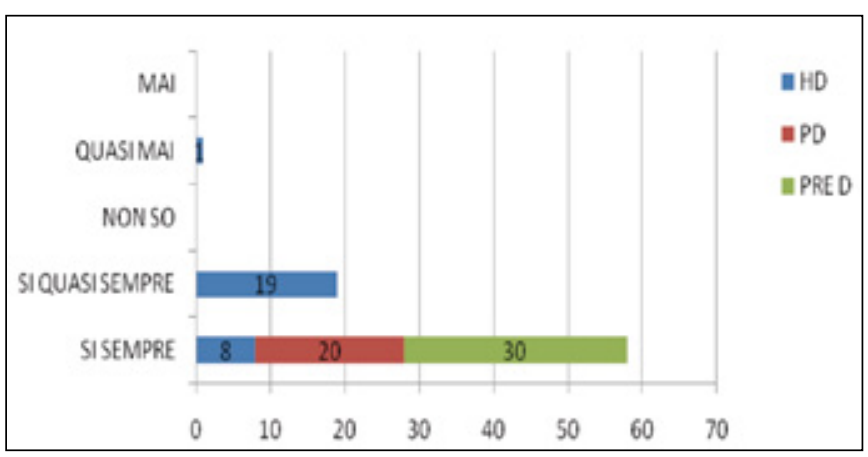

Fig. 1 - Quando l'infermiere le dà informazioni sulla sua malattia e sul suo trattamento è sempre chiaro e comprensibile?

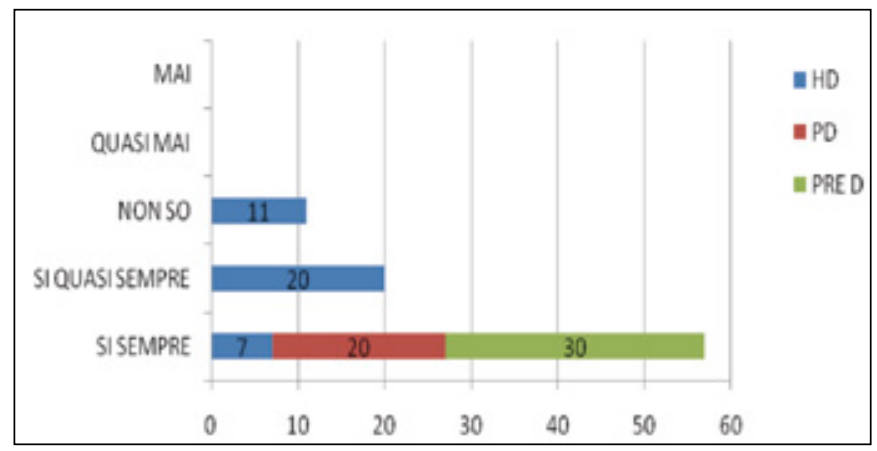

Fig. 3 - Quando ha necessità di parlare delle sue problematiche relative al suo stato di salute esiste un luogo idoneo al colloquio?

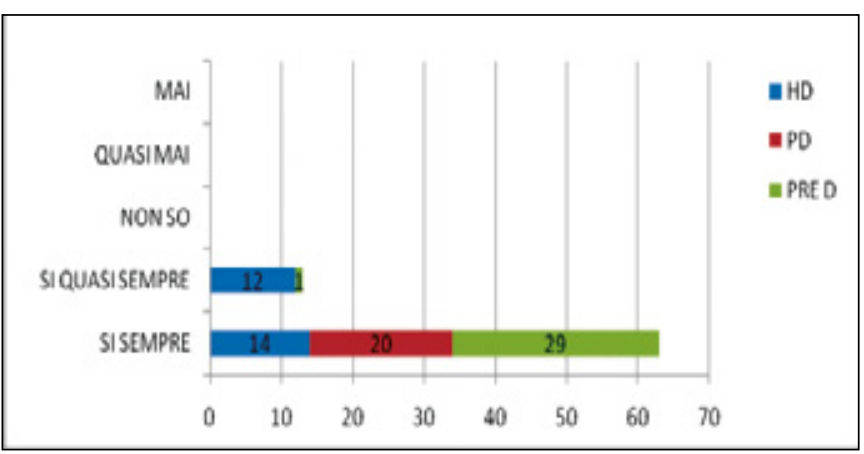

Fig. 5 - La comunicazione ricevuta dagli infermieri è chiara e adeguata alle sue capacità di comprensione?

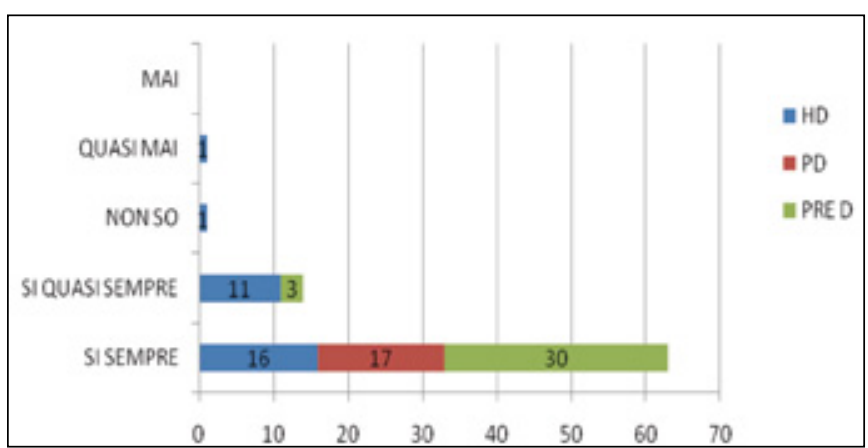

Fig. 7 - Si sente realmente compreso dell'infermiere quando parla della sua condizione attuale? 


\section{Discussione}

Obiettivo 1 - VERIFICARE L'ESISTENZA DI UN BUON RAPPORTO COMUNICATIVO TRA PAZIENTI E INFERMIERI:

Nell'unità operativa di dialisi peritoneale e predialisi si evince una buona disponibilità degli operatori a spiegare e a semplificare i termini medici, in modo tale da renderli comprensibili, relativamente alle capacità linguistiche e cognitive dei pazienti (domanda n. 1).

I pazienti che sono in predialisi e dialisi peritoneale $(100 \%)$ hanno risposto, infatti, "spesso", mentre i restanti pazienti in emodialisi hanno risposto "quasi sempre".

La risposta riguardante gli operatori e il loro prestare attenzione alla persona quando parla è stata perlopiù "quasi sempre" in emodialisi mentre, in dialisi peritoneale e in predialisi, $\mathrm{i}$ pazienti hanno risposto "sempre".

Questo dato abbastanza significativo corrisponde al tipo di rapporto che l'operatore sanitario in peritoneale instaura con il paziente, in quanto viene coinvolto in una dimensione più personale nel responsabilizzare il paziente nella presa in carico dello stato della malattia e della metodica dialitica.

Alla domanda sull'ascolto (n.4) gli infermieri di dialisi mettono in evidenza le difficoltà che hanno nel prestare attenzione anche se il valore è buono, prevale di più l'aspetto tecnico della seduta dialitica e quindi alcuni trascurano il resto.

Alla domanda n. 6 sulla privacy i pazienti di ambedue le unità danno soprattutto una risposta "sì sempre" e una piccola percentuale $(11 \%)$ risponde "quasi mai" e questo è legato perlopiù alla struttura ambientale, a causa della condivisione della stanza di emodialisi con più persone. Questo disagio è percepito anche dal personale infermieristico dell'emodialisi.

Obiettivo 2 - STABILIRE SE IL LIVELLO QUALITATIVO È PERCEPITO UGUALMENTE SIA DAGLI INFERMIERI CHE DAI PAZIENTI.

Le risposte ottenute dagli infermieri e dai pazienti risultano essere congruenti; pertanto, possiamo dire che il livello qualitativo della comunicazione è percepito in modo simile da entrambe le parti.

Obiettivo 3 - VERIFICARE SE LA COMUNICAZIONE INSTAURATA HA UN EFFETTO BENEFICO SULLA PSICOLOGIA DEL PAZIENTE, TALE DA PERMETTERGLI DI AFFRONTARE E SUPERARE EVENTUALI DISAGI SOCIALI/PSICOLOGICI/FAMILIARI APPORTATI DALLA MALATTIA E DAL TRATTAMENTO.

In questa realtà, il ruolo degli operatori è fondamentale, poiché essi devono comprendere i significati che i pazienti attribuiscono ai loro sintomi, vissuti e comportamenti e devono trovare, di volta in volta, le strategie relazionali più efficaci per tutelare lo svolgimento della seduta dialitica e, nei casi più gravi, la stessa vita del paziente. Questa necessità può creare nell'operatore un contrasto tra la sua formazione di base, certamente più attenta alle problematiche fisico-organiche, e la necessità di dover gestire una situazione molto problematica dal punto di vista relazionale, in cui, in assenza di adeguati strumenti psicologici, egli non può fare altro che subirla, con numerose conseguenze sul piano personale e relazionale. Infatti, tra gli operatori, è in atto un dibattito tra due orientamenti: coloro che ritengono che le problematiche psicologiche non siano così importanti ai fini del trattamento dialitico e che, quindi, minimizzano o, al più, delegano la loro gestione ai "tecnici" della psiche (psicologi, psichiatri) e coloro che invece ritengono necessario dotarsi di competenze psicologiche minime per poter gestire autonomamente il rapporto quotidiano con il paziente in emodialisi (4).

\section{Conclusioni}

Anche se la comunicazione assistenziale risulta formalmente ineccepibile (ipotesi 1), non altrettanto si può dire circa il livello empatico, soprattutto in emodialisi, rispetto alla dialisi peritoneale. Molti pazienti non sapevano dire se l'infermiere, in caso di disagi psicologici apportati dalla patologia, sapesse ascoltare con attenzione. I pazienti considerano comunque gli infermieri coinvolti nel loro stato di malattia, anche se gli operatori dichiarano di non riuscire a gestire bene gli eventuali coinvolgimenti emotivi. Gli infermieri in emodialisi sono consci di questo deficit comunicativo e, insieme ai malati, convivono nello stesso luogo senza entrare empaticamente in relazione. In dialisi peritoneale e predialisi, il gruppo infermieristico risulta più empatico nei confronti del paziente, si comprende che gli operatori tendono a prestare attenzione e il paziente si sente compreso. Inoltre, gli infermieri che seguono il paziente in predialisi e in dialisi peritoneale lo incontrano saltuariamente, in quanto i pazienti vengono a visita in ambulatorio con cadenza quasi sempre mensile oppure, in alcune realtà, effettuano la visita a domicilio, fuori, cioè, dall'ambiente ospedaliero che definisce i reciproci ruoli. Tale modalità relazionale è più impegnativa per gli operatori sanitari ma, alla lunga, è più gratificante ed efficiente, come testimoniato direttamente sia dallo staff che dai pazienti nei questionari raccolti. In emodialisi, il paziente lo si incontra tre volte alla settimana e ciò aumenta il "disagio psicologico" nel rapporto infermiere-paziente e, nell'operatore, possono comparire sentimenti di inadeguatezza e di "intrusione" da parte del paziente. L'infermiere può sentirsi continuamente giudicato "buono" o "cattivo", capace o incapace, con difficoltà nel regolare la "distanza terapeutica" nel lavoro assistenziale (4).

Il paziente in dialisi è un individuo che deve, in un certo senso, rinascere e crescere rapidamente per acquisire una posizione di matura collaborazione con l'equipe ed è necessario che i sanitari gli creino attorno un clima per quanto possibile ideale per questa crescita, acquisendo capacità di ascolto e di comprensione (5). L'ascolto è uno stimolo continuo, che aiuta a scoprire ogni giorno quanto si rischia di sbagliare quando non ci si sofferma a riconsiderare quello che qualsiasi persona è disposta a far notare. Sia in emodialisi che in dialisi peritoneale, alla base della scelta di una professione sanitaria vi è, seppure inconscia, la convinzione di arrivare a possedere strumenti efficaci contro la malattia e contro la morte; ne consegue che il paziente ideale è quello che fin dall'inizio è pienamente collaborante nella cura e che guarisce completamente. Nel caso particolare dell'infermiere in dialisi, è da sottolineare come l'avvento di questa tecnica sia stato vissuto magicamente come l'acquisizione di 
uno strumento onnipotente che avrebbe regalato la vita ai pazienti che prima erano accompagnati alla morte.

Ora è, però, nota la consapevolezza che la qualità della vita del paziente in dialisi è estremamente precaria e che, comunque, un buon adattamento a questa condizione di malattia cronica passa attraverso dolorose vicissitudini esistenziali che non conducono mai all'auspicata condizione di adattamento (6). L'ascolto è uno stimolo continuo, che ci aiuta a scoprire ogni giorno quanto si rischia di sbagliare quando non ci soffermiamo a riconsiderare quello che qualsiasi persona è disposta a farci notare.

\section{Riassunto}

Capire i bisogni di chi ci sta di fronte, accogliere, accettare ed essere autentici e saper ascoltare, comprendere e orientare le persone che chiedono aiuto sono capacità e abilità che si distinguono in chi fa counseling e ha scelto di percorrere una strada professionale difficile e impegnativa. Fondamentale di ogni progetto di aiuto è, quindi, una corretta comunicazione. In questo articolo, esamineremo quella tra infermiere e paziente in ambito dialitico. L'indagine è stata condotta dal 28 Maggio al 15 Giugno del 2012 con la distribuzione di un questionario ai pazienti uremici e al personale infermieristico operante nell'Unità Operativa di Emodialisi, Predialisi e Dialisi Peritoneale dell'Ospedale S. Orsola di Bologna.
Obiettivo della ricerca:

-Verificare l'esistenza di un rapporto comunicativo educativo tra malati e infermieri.

-Stabilire se il livello qualitativo è percepito ugualmente sia dagli infermieri che dai malati.

-Verificare se la comunicazione instaurata ha un effetto benefico sulla psicologia del paziente, tale da permettergli di affrontare e superare eventuali disagi sociali, psicologici e familiari apportati dalla malattia e dal trattamento.

Parole chiave: Counseling, Empatia, Relazione di aiuto, Paziente dializzato

Dichiarazione di conflitto di interesse: L'Autore dichiara di non avere conflitto di interessi.

Indirizzo degli Autori:

Maria Pia Zito

Infermiere Case Manager

U.O.Nefrologia, Dialisi e Trapianto

Azienda Ospedaliera di Bologna Policlinico S.Orsola-Malpighi

Via Massarenti 9

40138 Bologna

mariapiazito@tin.it

mariapia.zito@aosp.bo.it

\section{Bibliografia}

1. Zoia D. La relazione di aiuto nella comunicazione al malato uremico cronico. Tesi anno accademico 2001-2002.

2. Monformoso PG. Riflessione su l'arte clinica del prendersi cura. Edizione 2006, Editore APM Edizioni.

3. Nazzurri T. L'ascolto: il primo passo nella relazione con l'altro e come strumento relazionale in ambito dialitico presso l'Ospedale Policlinico S. Orsola Bologna. Tesi anno accademico 2011-2012.

4. Trabucco.G. Emodialisi e bisogni psicologici - Aspetti teorici, problemi psicologici-clinici. Pubblicato su Quaderni di Psico in: "Modelli di intervento dello psicologo in ospedale, confronto di esperienze. A cura di Roberto Ferretti e Maria Virginia Gentili. Disponibile su: http://www.sipsot.it/html/ricercafolder/ricerca ospedaliera/documenti/emodialisi/Emodialisi.htl.

5. Paris V. Vivere in dialisi: conoscere e sostenere il disagio psicosociale. Atti del Seminario "Vivere in dialisi: dalla qualità dei servizi alla qualità della vita". Roma 2004.

6. Lombardi M, Manfrina GM, Cerrai T, Dattolo P, Sisca C. Qualità della vita dell'uremico terminale. G Ital Nefrol 1994; 11 (4): 227-32.

\section{Testi consultati}

- Baldini M. Educare all'ascolto. La Scuola, Brescia 1988.

- Monformoso P, 2001. Istruzioni di counseling nell'assistenza, nella riabilitazione e nell'educazione. In: P. Monformoso, eds. I care... l'arte di prendersi cura secondi appunti, 2002. Busto Arsizio Edizione Privata 1-34.

- Monformoso P, 2002. Istruzioni di counseling nell'assistenza, nella riabilitazione e nell'educazione. In: P. Monformoso, eds. I care... l'arte di prendersi cura secondi appunti, 2002. Busto Arsizio Edizione Privata 10-42.

- Monformoso P. La relazione di aiuto nell'arte clinica CounselingVertici Network. Data di pubblicazione: 25/10/2004. 